\title{
Uniform Convergence Theorems of Boundary Solutions for Laplace's Equation
}

\author{
By \\ Yuusuke Iso*
}

\begin{abstract}
The numerical treatments for two-dimensional Laplace's equation with the Neumann condition are dealt with. The author gives a new numerical scheme for the boundary element methods to solve this problem. In this scheme, approximation for the domain is taken account of The author gives also the uniform convergence theorem for this new scheme, and proves it rigorously. This new scheme is both mathematical and practical.
\end{abstract}

\section{$\S 1$. Introduction}

The boundary element method (BEM) is one of the numerical methods to solve partial differential equations, esp. to solve boundary value problems of linear partial differential equations. In finite difference methods (FDM), we directly discretize our aimed differential equations. And in finite element methods (FEM), we discretize our aimed equations through variational forms. In both cases, the basic idea to construct numerical solutions is based on approximation of differential operators. But in BEM, the basic idea is somewhat different. We first convert the original linear differential equations into integral equations on the boundary of the domain. Then, we construct our aimed numerical solution through discretization of such integral equations. From this point of view, BEM may be classified as a numerical methods for integral equations.

In this paper, we discuss only on the Neumann problem of Laplace's equation. We show, in $\S 2$, reduction of this problem to integral equations on the boundary, which we call "boundary integral equation" in BEM. And we give two numerical schemes using the collocation method. The Scheme I shown in $\S 3$ is rather mathematical, but the Scheme II shown in $\S 5$ is both mathematical and practical. The uniform convergence theorems and their proofs are given in $\S 4$ and $\S 6$ respectively. And some remarks are given in $\S 7$.

By the way, the uniform convergence theorems for BEM using the collocation methods were also shown in Arnold-Wendland [1], and Arnold [2]. In their papers, they discretize the boundary integral equations without taking account

Received December 24, 1987.

* Research Institute for Mathematical Sciences, Kyoto University, Kyoto 606, Japan. 
of approximation for the boundaries. But we, in this paper, take account of such approximation when constructing the Scheme II.

Here we give some notations which are commonly used in this paper. Let $\Omega$ be a bounded domain in $\boldsymbol{R}^{2}$, and let $\Gamma$ be its boundary $\partial \Omega$. And let $x$ be spatial point $x=\left(x_{1}, x_{2}\right)$.

$C^{s}(\Omega)$ the set of $s$ times continuously differentiable functions defined on $\Omega$.

$C^{s}(\Gamma)$ the set of $s$ times continuously differentiable fnnctions defined on $\Gamma$.

$\|*\|_{\infty} \quad$ a maximum norm introduced into $C^{0}(\Gamma)$,

i.e. $\|f\|_{\infty}:=\max _{x \in \Gamma}|f(x)|$, where $f \in C^{0}(\Gamma)$.

$\|*\|_{2},\|*\|_{\infty}$ norm of $\boldsymbol{R}^{N}$ defined as follows ;

$$
\begin{aligned}
& \|x\|_{2}:=\left(\sum_{k=1}^{N} x_{k}^{2}\right)^{1 / 2} \\
& \|x\|_{\infty}:=\max _{k}\left(\left|x_{k}\right|\right)
\end{aligned}
$$

$\vec{n}_{z} \quad$ the unit outer normal vector at $z \in \Gamma$.

$\frac{\partial}{\partial n} \quad$ the outer normal derivative on $\Gamma$.

$m(l)$ the Lebesgue measure of a Jordan curve $l$.

$N(K) \quad$ the null space of an operator $K$

$D(K)$ the definition domain of an operator $K$

$\overline{z_{p} z_{q}} \quad$ a line segment between two points $z_{p}$ and $z_{q}$;

i. e. $\overline{z_{p} z_{q}}=\left\{z \mid z=\lambda z_{p}+(1-\lambda) z_{q}, 0 \leqq \lambda \leqq 1\right\}$

\section{§ . Boundary Integral Equation}

Let $\Omega$ be a bounded domain in $\boldsymbol{R}^{2}$, which satisfies the following assumptions.

\section{Assumption 2.1.}

(i) The boundary $\Gamma=\partial \Omega$ is of class $C^{3}$ 。

(ii) The curvature of the boundary is positive.

Here we consider the following Neumann problem of Laplace's equation :

$$
(N P) \begin{cases}\Delta u=0 & \text { in } \Omega \\ \frac{\partial}{\partial n} u=q & \text { on } \Gamma,\end{cases}
$$

where $q$ is a given function of class $C^{2}(\Gamma)$ which satisfies

$$
\int_{\Gamma} q(y) d \sigma_{y}=0 \text {. }
$$

Under above conditions, the problem $(N P)$ has a unique solution in $C^{2}(\bar{\Omega}) /$ \{const.\}. We, first, reduce this problem into integral equations on $\Gamma$.

Let $g(x, y)$ be a fundamental solution of two-dimensional Laplace's equation; 


$$
\text { i. e. } g(x, y)=-\frac{1}{2 \pi} \log |x-y| \text {. }
$$

If $u(x)$ is the smooth solution which satisfies (2.1), the following integral formula holds ;

$$
u(x)=-\int_{\Gamma} \frac{\partial}{\partial n_{y}} g(x, y) u(y) d \sigma_{y}+\int_{\Gamma} g(x, y) q(y) d \sigma_{y} \quad x \in \dot{\Omega} .
$$

Suppose the Dirichlet data of $(N P)$ is known, the solution of $(N P)$ can be expressed by (2.3). In order to calculate the Dirichlet data, we take the following limit of $(2.3)$ :

$$
\lim _{x \rightarrow z} u(x)=\lim _{x \rightarrow z}\left\{-\int_{\Gamma} \frac{\partial}{\partial n_{y}} g(x, y) u(y) d \sigma_{y}+\int_{\Gamma} g(x, y) q(y) d \sigma_{y}\right\}
$$

where $z$ is a point on $\Gamma$, and $x$ tends to $z$ in the inner normal direction at $z$. As $g(z, y)$ and $\left(\partial / \partial n_{y}\right) g(z, y)$ have singularities at $y=z$, we get the following formula ;

$$
u(z)=\frac{1}{2} u(z)-\mathrm{p} . \mathrm{v} \cdot \int_{\Gamma} \frac{\partial}{\partial n_{y}} g(z, y) u(y) d \sigma_{y}+\int_{\Gamma} g(z, y) q(y) d \sigma_{y}, \quad z \in \Gamma .
$$

Here "p.v." means the Cauchy principal value of an integral. Consequently we have

$$
\frac{1}{2} u(z)+\text { p.v. } \int_{\Gamma} \frac{\partial}{\partial n_{y}} g(z, y) u(y) d \sigma_{y}=\int_{\Gamma} g(z, y) q(y) d \sigma_{y}, \quad z \in \Gamma .
$$

This formula shows the analytic relation between the Dirichlet data and the Neumann data of Laplace's equation. We regard (2.4) as an integral equation for an unknown function $u(z)$, and call it a "boundary integral equation" in BEM.

Here we remark some properties of the kernel of this integral equation and give propositions to guarantee its solvability. Let $\vec{G}(x, y)$ be a vector valued function defined by

$$
\vec{G}(x, y):=\nabla_{y} g(x, y) .
$$

Hence we have $\left(\partial / \partial n_{y}\right) g(z, y)=\vec{G}(z, y) \cdot \vec{n}_{y}, y, z \in \Gamma$.

Proposition 2.1. Suppose Assumption_2.1 holds, and let us regard $\left(\partial / \partial n_{y}\right) g(z, y)$ as a function with respect to $y \in \Gamma$, whenever $z$ is fixed on $\Gamma$. Then, there exist positive constants $C$ and $C^{\prime}$ such that

$$
C \leqq \frac{\partial}{\partial n_{y}} g(z, y) \leqq C^{\prime} .
$$

Proof. As $\Gamma$ is a Jordan curve, we can parametrize it as follows;

$$
\Gamma=\left\{\left(y_{1}, y_{2}\right) \mid y_{1}=y_{1}(s), y_{2}=y_{2}(s),\left(\dot{y}_{1}\right)^{2}+\left(\dot{y}_{2}\right)^{2}=1, s \in I\right\},
$$

where we set an interval $I=\{s \in \boldsymbol{R} \mid-(1 / 2) m(\Gamma) \leqq s \leqq(1 / 2) m(\Gamma)\}$. Under Assump- 
tion 2.1, $y_{1}(s)$ and $y_{2}(s)$ belong to $C^{3}(I)$. We can set $z=\left(y_{1}(0), y_{2}(0)\right)$ in general. Under these preparations, we set a function $h(s)$ as follows;

$$
\begin{aligned}
h(s): & =\vec{G}(z, y) \cdot \vec{n}_{y} \\
& =-\frac{1}{2 \pi} \frac{\vec{p}(s) \cdot \vec{n}(s)}{\vec{p}(s) \cdot \vec{p}(s)},
\end{aligned}
$$

where $\vec{p}(s):=y-z=\left(y_{1}(s), y_{2}(s)\right)^{T}-\left(y_{1}(0), y_{2}(0)\right)^{T}$, and $\vec{n}(s):=\left(\dot{y}_{2}(s),-\dot{y}_{1}(s)\right)^{T}$. By convexity of $\Omega$, we have

$$
\vec{p}(s) \cdot \vec{n}(s)>0 \quad \text { for } \quad s \in I \text {. }
$$

Hence we have $h(s) \in C^{0}(I \backslash\{0\})$ and $h(s)<0$ for $s \in I$. Here we define $h^{\prime}(0)$ by $h(0):=\lim _{s \rightarrow 0} h(s)$. We are sufficient to show that $h(0)$ is finitely determined. For we come to regard $h(s)$ as a continuous function defined on a closed in terval $I$. And we can get the conclusion.

By the Taylor expansion near $s=0$, we have

$$
\begin{aligned}
& \vec{p}(s)=\vec{t}(0) s-\frac{1}{2} \kappa(0) \vec{n}(0) s^{2}+O\left(s^{3}\right), \\
& \vec{t}(s)=\vec{t}(0)-\kappa(0) \vec{n}(0) s+O\left(s^{2}\right), \\
& \vec{n}(s)=\vec{n}(0)+\kappa(0) \vec{t}(0) s+O\left(s^{2}\right),
\end{aligned}
$$

where $\vec{t}(s)=\left(\dot{y}_{1}(s), \dot{y}_{2}(s)\right)^{T}$ and $\kappa(s)$ denotes the curvature of $\Gamma$. Hence we have

$$
\lim _{s \rightarrow 0} h(s)=-\frac{1}{2 \pi} \cdot \frac{1}{2} \kappa(0)<0 \text {. }
$$

Remark 2.1. By this proposition, the integral kernel of (2.4) can be regarded as a continuous function and we may write

$$
\text { p.v. } \int_{\Gamma} \frac{\partial}{\partial n_{y}} g(z, y) u(y) d \sigma_{y}=\int_{\Gamma} \frac{\partial}{\partial n_{y}} g(z, y) u(y) d \sigma_{y} .
$$

We define an operator $K$ as follows;

$$
(K f)(z):=f(z)+\lim _{x \rightarrow z} \int_{\Gamma} \frac{\partial}{\partial n_{y}} g(x, y) f(y) d \sigma_{y},
$$

where $x \in \dot{Q}, z \in \Gamma, f \in C^{\circ}(\Gamma)$, and $x$ tends to $z$ in the inner normal direction at $z$.

Proposition 2.2 (see Kellogg [5; ch. 11])

$$
N(K)=\{\text { const. }\} \text {. }
$$

For the sake of convenience, we define $r(z)$ by

$$
r(z):=\int_{\Gamma} g(z, y) q(y) d \sigma_{y} \quad z \in \Gamma .
$$

Then, we can set up the problem to solve precisely.

Problem 2.1. Find a function $u \in C^{0}(\Gamma)$ such that 


$$
\begin{aligned}
& K u=r, \\
& \int_{\Gamma} u d \sigma=0 .
\end{aligned}
$$

Unique solvability of this problem is guaranteed by the next proposition.

Proposition 2.3. (see Kellogg [5; ch. 11]

The system of integral equations (2.9) and (2.10) has a unique solution $u \in C^{\circ}(\Gamma)$.

Under the assumption of regularity for the Neumann data $q$ and smoothness of $\Gamma$, the solution of (2.9) and (2.10) belongs to $C^{2}(\Gamma)$.

In this paper, our aim is to solve Problem 2.1 numerically and give the error estimates.

\section{§3. Discretization without Approximation of the Boundary}

In this section, we shall give a numerical scheme to solve Problem 2.1 without approximation of the boundary.

Let $\left\{z_{k}\right\}_{k=1}^{N}$ be a set of $N$ nodal points on $\Gamma$. We choose them so that they satisfy

$$
m\left(\widetilde{z}_{k} z_{k+1}\right)=\frac{1}{N} m(\Gamma) \quad 1 \leqq k \leqq N,
$$

where $z_{N+1}$ coincides with $z_{1}$ and $\widehat{z_{k} z_{k+1}}$ denotes a minor closed arc segment of $\Gamma$ between $z_{k}$ and $z_{k+1}$. We call an $\operatorname{arc} \widetilde{z_{k} z_{k+1}}$ a "boundary element", and denote it by $\Gamma_{k}$. Here we define a mesh size $h$ by

$$
h:=\frac{1}{N} m(\Gamma) .
$$

Let $v_{h}$ be an $N$-dimensional linear subspace of $C^{0}(\Gamma)$. The basis $\left\{\psi_{k}\right\}$ of $V_{h}$ must be chosen so that they satisfy the following assumptions.

\section{Assumption 3.1.}

(i) $\phi_{k} \in C^{0}(\Gamma), \quad \phi_{k}\left(z_{\jmath}\right)=\delta_{j, k}$ for $1 \leqq j, k \leqq N$,

(ii) $\operatorname{supp}\left(\phi_{k}\right)=\Gamma_{k-1} \cup \Gamma_{k}$ for $1 \leqq k \leqq N$, where $\Gamma_{0}=\Gamma_{N}$,

(iii) $\left.\phi_{k}\right|_{\Gamma_{\jmath}} \in C^{2}\left(\Gamma_{\jmath}\right)$ for $1 \leqq j, k \leqq N$,

(iv) $\sum_{k=1}^{N} \phi_{k}=1$,

(v) $\int_{\Gamma} \psi_{k} d \sigma=\int_{\Gamma} \psi_{j} d \sigma$ for $1 \leqq j, k \leqq N$.

In the case that $\Gamma$ is an one-dimensional smooth curve, we can easily construct an example of such base functions. Here $V_{h}$ is a linear span of $\left\{\psi_{k}\right\}_{k=1}^{N}$ 
We define an operator on $C^{0}(\Gamma)$ into $V_{h}$ by

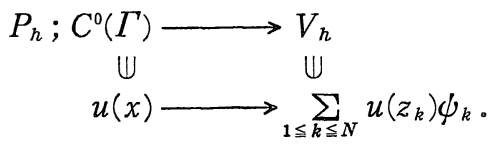

We call this operator $P_{h}$ a "collocation operator".

Proposition 3.1. Suppose $u(x) \in C^{2}(\Gamma)$. Then we have

$$
\left\|P_{h} u-u\right\|_{\infty} \leqq C h^{2},
$$

where $C$ is a positive constant which is independent of $h$.

Proof. Set $v:=P_{h} u-u$, then $v$ satisfies

$$
v \in C^{0}(\Gamma),\left.\quad v\right|_{\Gamma_{j}} \in C^{2}\left(\Gamma_{j}\right) \quad \text { for } \quad 1 \leqq j \leqq N .
$$

Namely $v$ is a piecewise $C^{2}$-function on $\Gamma$. By the Taylor expansion we have

$$
\max _{z \in \Gamma_{j}}|v(z)| \leqq C h^{2} \quad 1 \leqq j \leqq N,
$$

where $C$ is a positive constant which is independent of $h$. Hence we get our conclusion.

Furthermore, we define an operator $K_{h}$ on $V_{h}$ into $V_{h}$ by

$$
D\left(K_{h}\right)=V_{h}, \quad K_{h}:=P_{h} K .
$$

This operator corresponds to the discretized operator of the operator $K$.

Remark 3.1. We can identify the function space $V_{h}$ with $\boldsymbol{R}^{N}$. Let $u_{h}$ belong to $V_{h}$, then we have

$$
u_{h}=\sum_{k=1}^{N} a_{k} \psi_{k} .
$$

Therefore we can identify $\left(a_{1}, a_{2}, \cdots, a_{N}\right)^{T} \in \boldsymbol{R}^{N}$ with $u_{h}$. According to this identification rule, we can regard the operator $K_{h}$ as an $N \times N$ matrix. In this paper, we use the same notations for elements both of $V_{h}$ and of $\boldsymbol{R}^{N}$ as far as we are not in confusion.

Let $\left\{a_{i, j}\right\}_{1 \leq i \leq N \leq N}$ be a set of the elements of the matrix $K_{h}$, then they are expressed as follows:

$$
a_{i, j}=\left(P_{h} K \phi_{j}\right)\left(z_{i}\right) \quad 1 \leqq i, j \leqq N .
$$

Hence we have the following estimates. The next lemma plays an important role in the following discussion. Its proof is given in $\S 4$.

Lemma 3.1. Let $a_{i, j}(1 \leqq i, j \leqq N)$ be defined by (3.10). Then we have 
(i ) $-C_{1} h \leqq a_{i, j} \leqq-C_{2} h \quad i \neq j, 1 \leqq i, j \leqq N$,

(ii) $C_{3} \leqq a_{i, i} \leqq C_{4} \quad 1 \leqq i \leqq N$,

(iii) $\sum_{j=1}^{N} a_{\imath, j}=0$.

Here $C_{1}, C_{2}, C_{3}$, and $C_{4}$ are positive constants which are independent of $h$.

We get the next proposition immediately from this lemma.

Proposition 3.2. Let $K_{h}$ be the matrix defined above. Then we have

$$
\operatorname{rank}\left(K_{h}\right)=N-1 \text {. }
$$

Proof. By (3.13), it is clear that $\operatorname{rank}\left(K_{h}\right) \leqq N-1$. Let $K_{h}^{\prime}$ be the first minor matrix of $K_{h}$;

$$
\text { i. e. } K_{h}^{\prime}=\left(a_{i, j}\right) \underset{1 \leqq i \leqq N-1}{1 \leqq j \leqq N-1} \text {. }
$$

From (3.11), (3.12), and (3.13), this matrix $K_{h}^{\prime}$ is a diagonally dominant matrix. Hence we have $\operatorname{rank}\left(K_{h}^{\prime}\right)=N-1$. Thus we get our conclusion. (q. e. d.)

Let $\mu=\left(\mu_{1}, \mu_{2}, \cdots, \mu_{N}\right)^{T}$ be an element of $\boldsymbol{R}^{N}$ such that

$$
\mu_{N} \neq 0, \quad K_{h}{ }^{T} \mu=0 .
$$

Proposition 3.2 enables us to find such an element $\mu \in \boldsymbol{R}^{N}$. Using $\mu$, we give discretization of the function $r(z)$, which is defined by (2.8), as follows :

$$
\begin{aligned}
r_{h}^{k} & :=r\left(z_{k}\right) \quad 1 \leqq k \leqq N-1, \\
r_{h}^{N} & :=-\frac{1}{\mu_{N}} \sum_{k=1}^{N-1} \mu_{k} r_{h}^{k} .
\end{aligned}
$$

Under above preparations, we set up the following problem which corresponds to the discretized problem of Problem 2.1.

Problem 3.1 (Scheme I). Find a solution $u_{h} \in \boldsymbol{R}^{N}$ such that

$$
\begin{gathered}
K_{h} u_{h}=r_{h}, \\
\sum_{k=1}^{N} u_{h}^{k}=0,
\end{gathered}
$$

where $u_{h}=\left(u_{h}^{1}, u_{h}^{2}, \cdots, u_{h}^{N}\right)^{T}, r_{h}=\left(r_{h}^{1}, r_{h}^{2}, \cdots, r_{h}^{N}\right)^{T}$.

From Proposition 3.2 and the definitions for $\mu$ and $r_{h}$, we can get the next theorem which ensures unique solvability of Problem 3.1.

Theorem 3.1. There exists a unique solution $u_{h} \in \boldsymbol{R}^{N}$ of the equations (3.17) and (3.18). 


\section{§4. Covergence Theorem for Scheme I}

Let $u$ be the solution of Problem 2.1, and let $u_{h}$ be the solution of Problem 3.1. According to Remark 3.1, we can regard $u_{h}$ as an element of $V_{h}$. That is, $u_{n}$ can be regarded as a continuous function on $\Gamma$. In this section, we establish the uniform convergence theorem for Scheme I through estimation of $\left\|P_{h} u-u_{h}\right\|_{\infty}$. We start from the proof of Lemma 3.1.

Proof of Lemma 3.1. From Proposition 2.1, we have

$$
-C_{1} h \leqq \int_{\Gamma_{j-1} \cup \Gamma_{j}} \vec{G}\left(z_{\imath}, y\right) \cdot \vec{\imath}{ }_{y} \psi_{\jmath}(y) d \sigma_{y} \leqq-C_{2} h, \quad 1 \leqq i, j \leqq N,
$$

where $C_{1}$ and $C_{2}$ are positive constants which are independent of $h$. Furthermore we have

$$
a_{i, \imath}=\frac{1}{2}+\int_{\Gamma_{i-1} \cup \Gamma_{i}} \vec{G}\left(z_{\imath}, y\right) \vec{n}_{y} \psi_{\imath}(y) d \sigma_{y}, \quad 1 \leqq i \leqq N .
$$

Hence we get (3.11) and (3.12). On the other hand, we have, from (3.6),

$$
\int_{\Gamma} \frac{\partial}{\partial n_{y}} g(x, y)\left(\sum_{j=1}^{N} \psi_{J}(y)\right) d \sigma_{y}=-1 \quad \text { for } \quad x \in \dot{\Omega} .
$$

Thus we have

$$
\begin{aligned}
\sum_{j=1}^{N} a_{i, \jmath} & =1+\lim _{x \rightarrow z_{i}} \int_{\Gamma} \frac{\partial}{\partial n_{y}} g(x, y)\left(\sum_{\jmath=1}^{N} \psi_{\jmath}(y)\right) d \sigma_{y} \\
& =0
\end{aligned}
$$

In order to estimate $\left\|P_{h} u-u_{h}\right\|_{\infty}$, we estimate $K_{h}\left(P_{h} u-u_{h}\right)$. We decompose it into the following form;

$$
K_{h}\left(P_{h} u-u_{h}\right)=P_{h} K\left(P_{h} u-u\right)+\left(P_{h} r-r_{h}\right) .
$$

Let $e_{h}$ be an element of $V_{h}$ defined by

$$
e_{h}:=P_{h} u-u_{h} \text {. }
$$

We are sufficient to estimate $\left\|e_{h}\right\|_{\infty}\left(=\left\|e_{h}\right\|_{\infty}\right)$. We prepare the next propositions for our aim. Let $K_{h}^{\prime}$ be the first minor matrix of the matrix $K_{h}$;

$$
\text { i. e. } K_{h}^{\prime}=\left(a_{i, \jmath}\right)_{1 \leq i \leq N-1} \text {. }
$$

As is mentioned before, $K_{h}^{\prime}$ is a regular matrix. It has the next estimate.

Proposition 4.1. For a sufficiently large integer $N$, we have

$$
\left\|K_{h}^{\prime-1}\right\|_{\infty} \leqq C \frac{1}{h},
$$

where $C$ is a positive constant which is independent of $h$. 
Proof. The matrix $K_{h}^{\prime}$ is diagonally dominant and can be decomposed into the following form;

$$
K_{h}^{\prime}=D_{h}^{\prime}\left(I+D_{h}^{\prime-1}\left(K_{h}^{\prime}-D_{h}^{\prime}\right)\right),
$$

where the matrix $D_{h}^{\prime}$ is a diagonal matrix defined by

$$
D_{h}^{\prime}=\operatorname{diag}\left(a_{1,1}, a_{2,2}, \cdots \cdots, a_{N-1, N-1}\right) .
$$

And obviously the matrix $D_{h}^{\prime}$ is a regular matrix. Using the results of Lemma 3.1, we have

$$
\begin{aligned}
\left\|D_{h}^{\prime-1}\left(K_{h}^{\prime}-D_{h}^{\prime}\right)\right\|_{\infty} & =\max _{i} \sum_{\substack{j=1 \\
j \neq i}}^{N-1} \frac{\left|a_{i, j}\right|}{\left|a_{i, i}\right|} \\
& =\max _{i} \frac{a_{i, i}+a_{\imath, N}}{a_{\imath, i}} \\
& \leqq 1-C h,
\end{aligned}
$$

where $C$ is a positive constant which is independent of $h$. By the technique of Neumann's series, we get for a sufficiently large integer $N$ (i. e. for a sufficiently small positive number $h$ ),

$$
\begin{aligned}
\left\|K_{h}^{\prime-1}\right\|_{\infty} & =\left\|D_{h}^{\prime-1}\right\|_{\infty} \cdot\left\|\left(I+D_{h}^{\prime-1}\left(K_{h}^{\prime}-D_{h}^{\prime}\right)\right)^{-1}\right\|_{\infty} \\
& \leqq\left\|D_{h}^{\prime-1}\right\|_{\infty} \cdot \frac{1}{1-(1-C h)} \\
& \leqq C^{\prime} \cdot \frac{1}{h} .
\end{aligned}
$$

Proposition 4.2. Let $u$ be the solution of (2.9) and (2.10), then ue have

$$
\left\|P_{h} K\left(P_{h} u-u\right)\right\|_{\infty} \leqq C h^{2},
$$

where $C$ is a positive constant which is independent of $h$.

Proof. Here we remark that $u$ belongs to $C^{2}(\Gamma)$. (See Remark 2.2.) By (2.7), we have

$$
\begin{aligned}
& \left\|P_{h} K\left(P_{h} u-u\right) \mid\right\|_{\infty} \\
& \leqq\left\|P_{h} u-u\right\|_{\infty}+\max _{i}\left(\lim _{x \rightarrow z_{i}}\left|\int_{\Gamma} \frac{\partial}{\partial n_{y}} g(x, y)\left(P_{h} u-u\right)(y) d \sigma_{y}\right|\right) .
\end{aligned}
$$

From (4.1), we have

$$
\begin{aligned}
& \lim _{x \rightarrow z_{i}}\left|\int_{\Gamma} \frac{\partial}{\partial n_{y}} g(x, y)\left(P_{h} u-u\right)(y) d \sigma_{y}\right| \\
& \leqq|| P_{h} u-u\|\|_{\infty} \cdot \lim _{x \rightarrow z_{i}}\left|\int_{\Gamma} \frac{\partial}{\partial n_{y}} g(x, y) d \sigma_{y}\right| \\
& =\mid\left\|P_{h} u-u\right\|_{\infty} .
\end{aligned}
$$

Hence we have 


$$
\left\|P_{h} K\left(P_{h} u-u\right)\right\|_{\infty} \leqq 2\left\|P_{h} u-u\right\|_{\infty} .
$$

On the other hand, from Proposition 3.1, we have

Therefore we are to get

$$
\left\|P_{h} u-u\right\|_{\infty} \leqq C h^{2} \text {. }
$$

$$
\left\|P_{h} K\left(P_{h} u-u\right)\right\|_{\infty} \leqq C h^{2},
$$

where $C$ is a positive constant which is independent of $h$.

Proposition 4.3. Let $u$ be the solution of (2.9) and (2.10), then we have

$$
\left|\int_{\Gamma}\left(P_{h} u-u_{h}\right) d \sigma\right| \leqq C h^{2},
$$

where $C$ is a positive constant which is independent of $h$.

Proof. From (3.7) and (3.19), we have

$$
\int_{\Gamma} u_{h} d \sigma=0
$$

Hence we have

From (4.6), we have

$$
\begin{aligned}
\left|\int_{\Gamma}\left(P_{h} u-u_{h}\right) d \sigma\right| & =\left|\int_{\Gamma}\left(\left(P_{h} u-u\right)+u-u_{h}\right) d \sigma\right| \\
& \leqq \int_{\Gamma}\left|P_{h} u-u\right| d \sigma \\
& \leqq m(\Gamma) \cdot \mid\left\|P_{h} u-u\right\|_{\infty} .
\end{aligned}
$$

$$
\left|\int_{\Gamma}\left(P_{h} u-u_{h}\right) d \sigma\right| \leqq C h^{2},
$$

where $C$ is a positive constant which is independent of $h$.

Under above preparations, we can prove the next theorem, which guarantees uniform convergence for "Scheme I".

Theorem 4.1. Let $e_{h}$ be an element of $V_{h}$ defined by (4.2). Then we have

$$
\left\|e_{h}\right\|_{\infty} \leqq C h,
$$

where $C$ is a positive constant which is independent of $h$.

Proof. Define two matrices $S$ and $T$ by

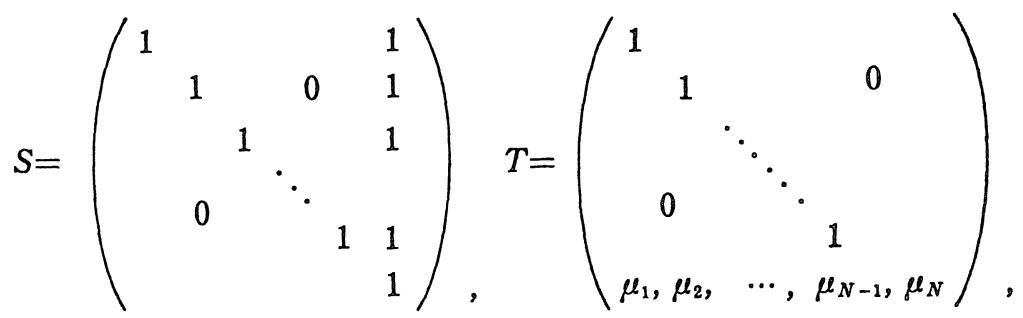


where $\mu_{1}, \cdots \mu_{N}$ are defined by (3.15). Then we have

$$
S^{-1}=\left(\begin{array}{ccccc}
1 & & & & -1 \\
& 1 & & 0 & -1 \\
& & \ddots & \vdots \\
& 0 & & & 1 \\
& & & & 1
\end{array}\right),
$$

and we have

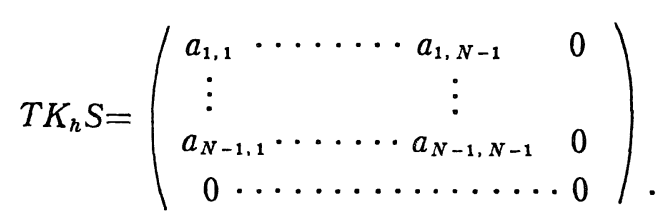

Operating two matrices $S$ and $T$ to $e_{h}$, we have

$$
T K_{h} S S^{-1} e_{h}=T P_{h} K\left(P_{h} u-u\right)+T\left(P_{h} r-r_{h}\right) .
$$

Let us set the right hand side of (4.9) in the following form;

$$
T P_{h} K\left(P_{h} u-u\right)+T\left(P_{h} r-r_{h}\right)=\left(t_{h}^{1}, t_{h}^{2}, \cdots, t_{h}^{N-1}, *\right)^{T},
$$

then, by (4.3), we have

$$
\mid t_{h}^{k} ! \leqq C h \quad \text { for } \quad 1 \leqq k \leqq N-1 .
$$

From (4.9), (4.10) and (4.11), we have

$$
e_{h}^{k}-e_{h}^{N}=t_{h}^{k} \quad \text { for } \quad 1 \leqq k \leqq N-1 .
$$

Furthermore from Assumption 3.1 and (4.7), we have

Set again

$$
e_{h}^{1}+e_{h}^{2}+\cdots \cdots+e_{h}^{N}=O(h) .
$$

then we have

$$
t_{h}^{\mathrm{V}}:=e_{h}^{1}+e_{h}^{2}+\cdots \cdots+e_{h}^{N},
$$

From (4.9)-(4.13), we have

$$
t_{h}^{N}=O(h)
$$

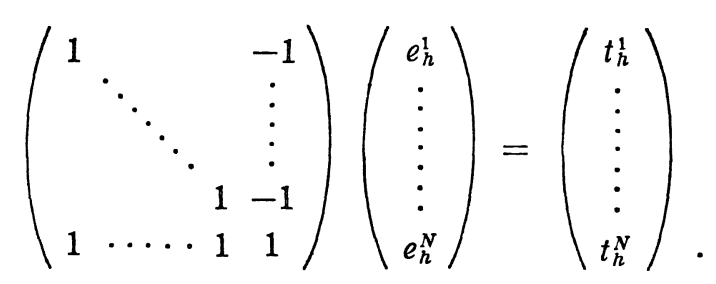

Here $t_{h}^{h}$ satisfies 


$$
\left|t_{h}^{k}\right| \leqq C h \quad \text { for } \quad 1 \leqq k \leqq N,
$$

where $C$ is a positive constant which is independent of $h$. Applying the method of Gauss' elimination to (4.14), we are to get

$$
\left(\begin{array}{ccccc}
1 & & & \\
& \ddots & & 0 & \\
& & \ddots & & \\
& 0 & & 1 & \\
& & & & N
\end{array}\right)\left(\begin{array}{c}
e_{h}^{1} \\
\vdots \\
\vdots \\
e_{h}^{N-1} \\
e_{h}^{N}
\end{array}\right)=\left(\begin{array}{l}
t_{h}^{1} \\
\vdots \\
\vdots \\
t_{h}^{N-1} \\
t_{h}^{N}-t_{h}^{1}-\cdots-t_{h}^{N-1}
\end{array}\right) .
$$

Hence we have

and successively we can get

$$
\begin{aligned}
\left|e_{h}^{N}\right| & \leqq \frac{1}{N} \sum_{k=1}^{N}\left|t_{h}^{k}\right| \\
& \leqq C h
\end{aligned}
$$

$$
\begin{aligned}
\left|e_{h}^{k}\right| & \leqq\left|e_{h}^{k}-e_{h}^{N}\right|+\left|e_{h}^{N}\right| \\
& \leqq C h
\end{aligned}
$$

for $1 \leqq k \leqq N-1$. Then we get the conclusion.

(q. e.d.)

This theorem guarantees uniform convergence for the Scheme I. But this numerical scheme is not ment for practical uses. Constructing numerical solutions by computers, we always approximate the boundary by some polygonal lines. This approximation yields another kind of discretization errors which are not taken account of in this section.

\section{$\S 5$. Construction of Scheme II-Discretization with Approximation of the Boundary}

Let $\left\{z_{2 k-1}\right\}_{k=1}^{N}$ be a set of $N$ nodal points on $\Gamma$. Let $\gamma_{j}$ be a closed line segment between two points $z_{2 j-1}$ and $z_{2 j+1}$ for $1 \leqq j \leqq N$; i. e. $\gamma_{j}=\bar{z}_{2 j-1} z_{2 j+1}$. And let $\Gamma_{j}$ be a closed minor arc segment of $\Gamma$ between $z_{2 j-1}$ and $z_{2 j+1}$; i. e. $\Gamma_{j}=\overparen{z_{2 j-1}} z_{2 j+1}$. And let $h$ be defined by

$$
h=\max _{j} m\left(\gamma_{j}\right) \text {. }
$$

Moreover we denote a polygon $\bigcup_{1 \leqq j \leqq N} \gamma_{j}$ by $\tilde{\Gamma}$. This set of nodal points must be chosen so that they satisfy the following assumption.

Assumption 5.1. There exists a positive constant $M$ such that

$$
\frac{\max m\left(\gamma_{j}\right)}{\min m\left(\gamma_{j}\right)} \leqq M
$$

where $M$ is independent of $h$. 
And let $\nu_{j}$ be a unit outer normal vector of $\tilde{\Gamma}$ on $\gamma_{j}$. Furthermore we define a vector $\vec{\eta}_{j}$ as follows:

$$
\vec{\eta}_{j}=\frac{m\left(\gamma_{j}\right) \nu_{j-1}+m\left(\gamma_{j-1}\right) \nu_{j}}{\left\|m\left(\gamma_{j}\right) \nu_{j-1}+m\left(\gamma_{j-1}\right) \nu_{j}\right\|_{2}} \quad \text { for } \quad 1 \leqq j \leqq N
$$

where $\nu_{0}$ and $\gamma_{0}$ coincide with $\nu_{N}$ and $\gamma_{N}$ respectively.

Proposition 5.1. Under Assumption 2.1, we have

$$
\left\|\vec{\eta}_{j}-\vec{n}_{z_{2 j-1}}\right\|_{\infty} \leqq C h^{2},
$$

where $C$ is a positive constant which is independent of $h$.

Proof. It is sufficient to prove for the case of $j=1$. We parametrize the boundary $\Gamma$ in the same way in the proof of Proposition 2.1. Here we can assume in general that

$$
\begin{aligned}
& z_{1}=\left(y_{1}(0), y_{2}(0)\right)=(0,0), \\
& \left(\dot{y}_{1}(0), \dot{y}_{2}(0)\right)=(1,0) .
\end{aligned}
$$

In this case, it is clear that

$$
\vec{n}_{z_{1}}=(0,-1)^{T}
$$

Furthermore we set

$$
\begin{aligned}
& z_{3}=\left(y_{1}\left(s_{+}\right), y_{2}\left(s_{+}\right)\right), \\
& z_{2 N-1}=\left(y_{1}\left(s_{-}\right), y_{2}\left(s_{-}\right)\right),
\end{aligned}
$$

where $s_{-}<0<s_{+}$, and they satisfy $\max \left(\left|s_{-}\right|, s_{+}\right) \leqq C h$, where $C$ is a positive constant which is independent of $h$. Under above preparations, we get

$$
\begin{aligned}
& \nu_{1}=\frac{1}{1_{+}}\left(y_{2}\left(s_{+}\right),-y_{1}\left(s_{+}\right)\right)^{T}, \\
& \nu_{N}=\frac{1}{1_{-}}\left(-y_{2}\left(s_{-}\right), y_{1}\left(s_{-}\right)\right)^{T}
\end{aligned}
$$

where $1_{+}=\left(\left(y_{1}\left(s_{+}\right)\right)^{2}+\left(y_{2}\left(s_{+}\right)\right)^{2}\right)^{1 / 2}$, and $1_{-}=\left(\left(y_{1}\left(s_{-}\right)\right)^{2}+\left(y_{2}\left(s_{-}\right)\right)^{2}\right)^{1 / 2}$.

By (5.1), we get

$$
\vec{\eta}_{1}=\frac{1_{+} \nu_{N}+1_{-\nu_{1}}}{\left\|1_{+} \nu_{N}+1_{-} \nu_{1}\right\|_{2}}
$$

By the Taylor expansion, we have

$$
\begin{aligned}
& \nu_{1}=(0,-1)^{T}+s_{+} \kappa(0)(1,0)^{T}+O\left(h^{2}\right), \\
& \nu_{N}=(0,-1)^{T}+s_{-} \kappa(0)(1,0)^{T}+O\left(h^{2}\right),
\end{aligned}
$$

where $\kappa(0)$ denotes the curvature of $\Gamma$ at $s=0$. From (5.9) and (5.10), we get

$$
\vec{\eta}_{1}=(0,-1)^{T}+O\left(h^{2}\right) \text {. }
$$


Thus by (5.5) and (5.11), we are to get

$$
\left\|\vec{\eta}_{1}-\vec{n}_{z_{1}}\right\|_{\infty} \leqq C h^{2} \text {, }
$$

where $C$ is a positive constant which is independent of $h$.

(q.e.d.)

Let $z_{2 k}$ be the middle point of the line segment $\gamma_{k}$ for $1 \leqq k \leqq N$, and let $\left\{z_{2 k}\right\}_{k=1}^{N}$ be another set of $N$ nodal points on $\tilde{\Gamma}$. Let us denote a point on $\tilde{\Gamma}$ by $\tilde{z}$. Here $\tilde{z}$ is chosen so that $\tilde{z} \in \overline{z_{2 j} z_{2 j-1}}$ in convenience. Let us set $\lambda:=m\left(\overline{\left.z_{2,} \tilde{z}\right)}\right.$. Then we define a vector $\vec{m}_{\tilde{z}}$ as follows (see Fig. 1);

$$
\vec{m}_{\tilde{z}}:=\left(1-\frac{2 \lambda}{m\left(\gamma_{j}\right)}\right) \nu_{j}+\frac{2 \lambda}{m\left(\gamma_{j}\right)} \vec{\eta}_{j} .
$$

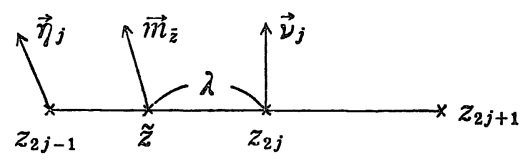

(Fig. 1)

Roughly speaking, $\vec{m}_{z}$ is defined as the linearly interpolated vector of the vectors $\left\{\vec{\eta}_{\jmath}\right\}$ and $\left\{\nu_{\jmath}\right\}$. By this rule, we have defined the vector $\vec{m}_{\tilde{z}}$ throughout on $\tilde{\Gamma}$, and we call it a 'pseudo-normal' vector of $\tilde{\Gamma}$.

Next we choose a set of piecewise-linear functions $\left\{\tilde{\phi}_{k}\right\}_{k=1}^{2 N}$ on the polygon $\tilde{\Gamma}$ so that they satisfy the following assumptions.

\section{Assumption 5.2.}

(i) $\tilde{\phi}_{k} \in C^{0}(\tilde{\Gamma})$ for $1 \leqq k \leqq 2 N$.

(ii) $\tilde{\phi}_{k}\left(z_{\jmath}\right)=\delta_{k, j}$ for $1 \leqq j, k \leqq 2 N$.

(iii) $\tilde{\phi}_{k}$ is a linear function on the each line segment $\bar{z}_{j} z_{j+1}$ for $1 \leqq j, k \leqq 2 N$.

Using these 'small' piecewise-linear functions $\left\{\tilde{\phi}_{k}\right\}_{k=1}^{2 N}$, we construct another set of piecewise-linear functions. Let us define 'large' piecewise-linear functions as follows;

$$
\tilde{\phi}_{j}=\tilde{\phi}_{j}+\frac{1}{2}\left(\tilde{\phi}_{j-1}+\tilde{\phi}_{j+1}\right),
$$

for $1 \leqq j \leqq 2 N$. Let $\tilde{V}_{h}^{1}$ and $\tilde{V}_{h}^{2}$ be finite dimensional function spaces on $\tilde{\Gamma}$ defined by

$$
\begin{aligned}
& \tilde{V}_{h}^{1}=\text { linear span of }\left\{\tilde{\psi}_{1}, \tilde{\psi}_{3}, \cdots, \tilde{\psi}_{2 N-1}\right\}, \\
& \tilde{V}_{h}^{2}=\text { linear span of }\left\{\tilde{\psi}_{2}, \tilde{\psi}_{4}, \cdots, \tilde{\psi}_{2 N}\right\} .
\end{aligned}
$$

And let $\tilde{P}_{h}^{1}$ be a collocation operator on $C^{0}(\Gamma)$ into $\tilde{V}_{h}^{1}$ defined by

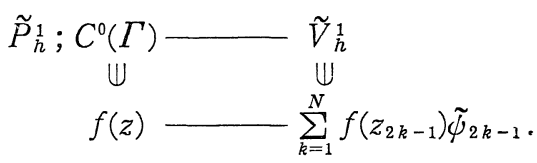


Under above preparations, we discretize the operator $K$. As is mentioned in Remark 3.1, we are sufficient to give the all elements of the matrix which corresponds to the discretized operator $\tilde{K}_{h}$.

Set

$$
\alpha_{\imath, j}:=\delta_{\imath, j}+\lim _{x \rightarrow z_{2 i}} \int_{\widetilde{\Gamma}} \vec{G}(x, \tilde{y}) \cdot \vec{m}_{\tilde{y}} \tilde{\psi}_{2,} d \sigma_{\tilde{y}}
$$

for $1 \leqq i, j \leqq N$. And set

$$
\zeta_{\imath}:=\sum_{j=1}^{N} \alpha_{\imath, j} \quad \text { for } \quad 1 \leqq i \leqq N .
$$

Furthermore set

$$
\iota_{2, \jmath}:=\alpha_{\imath, \jmath}-\frac{1}{N} b_{i} \quad \text { for } 1 \leqq i, j \leqq N .
$$

Let us define the matrix $\widetilde{K}_{h}$ by $\widetilde{K}_{h}:=\left(c_{\imath, \jmath}\right)$. Then the following estimates can be got.

Proposition 5.2. Let $\left\{b_{2}\right\}$ be defined by (5.17). Then we have

$$
\left|b_{2}\right| \leqq C h^{2} \quad \text { for } 1 \leqq i \leqq N \text {, }
$$

where $C$ is a positive constant which is independent of $h$.

Lemma 5.1. Let $N$ be a sufficiently large integer. Then, for the eleinents of the matrix $\tilde{K}_{h}$ defined by (5.18), we have the following estimates:

(i) $-C_{1} h \leqq c_{i, j} \leqq-C_{2} h$ for $1 \leqq i, j \leqq N$ and $i \neq j$.

(ii) $C_{3} \leqq c_{i, i} \leqq C_{4}$ for $1 \leqq i \leqq N$,

(iii) $\sum_{j=1}^{N} c_{i, j}=0$.

where $C_{1}, C_{2}, C_{3}$ and $C_{4}$, are positive constants which are independent of $h$.

Their proofs are given in the next section. This lemma plays the same role with Lemma 3.1. And the next proposition follows immediately from this lemma.

Proposition 5.3. Let $\tilde{K}_{h}$ be the above defined matrix, then we have

$$
\operatorname{rank}\left(\tilde{K}_{h}\right)=N-1 \text {. }
$$

Next let us define a function $\tilde{r}(\tilde{z})$ by

$$
\tilde{r}(\tilde{z})=\int_{\tilde{\Gamma}} g(\tilde{z}, \tilde{y}) \tilde{P}_{h}^{1} q d \sigma_{\tilde{y}} .
$$

And let $\tilde{\mu}$ be an element of $\boldsymbol{R}^{N}$ such that $\tilde{\mu}_{N} \neq 0, \tilde{K}_{h}{ }^{T} \tilde{\mu}=0$. Using this vector, we discretize $\tilde{r}(\tilde{z})$ as follows: 


$$
\begin{aligned}
& \tilde{r}_{h}^{k}=\tilde{r}\left(z_{2 k}\right) \quad \text { for } \quad 1 \leqq k \leqq N-1, \\
& \tilde{r}_{h}^{N}=-\frac{1}{\tilde{\mu}_{N}} \sum_{k=1}^{N-1} \tilde{\mu}_{k} \tilde{r}_{h}^{h},
\end{aligned}
$$

where $\tilde{\mu}=\left(\tilde{\mu}_{1}, \tilde{\mu}_{2}, \cdots, \tilde{\mu}_{N}\right)^{T}$. Hence we set up the next approximating problem for Problem 2.1.

Problem 5.1 (Scheme II). Find a solution $u_{h} \in \boldsymbol{R}^{N}$ such that

$$
\begin{aligned}
& \left(K_{h}^{\prime}\right) \tilde{u}_{h}=\tilde{r}_{h}, \\
& \sum_{k=1}^{N} d_{k} \tilde{u}_{h}^{k}=0,
\end{aligned}
$$

where $\tilde{r}_{h}=\left(\tilde{r}_{h}^{1}, \tilde{r}_{h}^{0}, \cdots, \tilde{r}_{h}^{N}\right)^{T}$, and $\left\{d_{k}\right\}$ are defined by

$$
d_{k}=\int_{\widetilde{\Gamma}} \tilde{\psi}_{2 k} d \sigma \quad \text { for } 1 \leqq k \leqq N .
$$

We call it, in this paper, "Scheme II". Considering the basic idea to construct this scheme, we can easily get the theorem for unique solvability of the above problem.

Theorem 5.1. There exists a unique solution $\tilde{u}_{h} \in \boldsymbol{R}^{N}$ of equations (5.26) and (5.27).

\section{§6. Convergence Theorem for Scheme II}

Prior to the main discussion, we define another pseudo-normal vector of $\tilde{\Gamma}$. Let $\vec{m}_{\tilde{z}}^{\prime}$ be a vector defined by replacement of $\vec{\eta}_{j}$ with $\vec{n}_{z_{2 j-1}}$ in (5.12):

$$
\text { i. e. } \vec{m}_{\tilde{z}}^{\prime}:=\left(1-\frac{2 \lambda}{m\left(\gamma_{j}\right)}\right) \nu_{j}+\frac{2 \lambda}{m\left(\gamma_{j}\right)} \vec{n}_{z_{2 j-1}} \text {, }
$$

where $\tilde{z} \in{\overline{z_{2 j-1}}}_{2 j}$ and $\lambda=m\left(\overline{z_{2 j} \tilde{z}}\right)$. Obviously we have

$$
\vec{n}_{z_{2 j-1}}^{\prime}=\vec{n}_{z_{2 j-1}}, \quad \vec{m}_{z_{2 j}}^{\prime}=\nu_{j} \text {. }
$$

Here the next proposition holds.

Proposition 6.1. Let $v(\tilde{y})$ be a function which belongs to $C^{0}(\tilde{\Gamma})$. Then, we have

$$
\left|\int_{\gamma_{j}} \vec{G}\left(z_{2 k}, \tilde{y}\right) \cdot\left(\vec{m}_{\tilde{y}}-\vec{m}_{\tilde{y}}^{\prime}\right) v(\tilde{y}) d \sigma_{\tilde{y}}\right| \leqq C h^{3}
$$

for $1 \leqq j, k \leqq N$, where $C$ is a positive constant which is independent of $h, j$ and $k$.

Proof. Set

$$
H_{k}(\tilde{y}):=\vec{G}\left(z_{2 k}, \tilde{y}\right) \cdot\left(\vec{m}_{\tilde{y}}-\vec{m}_{\tilde{y}}^{\prime}\right) \quad \text { for } \quad 1 \leqq k \leqq N .
$$

Considering (5.12) and (6.1), we can regard a function $H_{k}(\tilde{y})$ as a continuous 
function on $\tilde{\Gamma}$. Moreover this function is piecewise-differentiable: $H_{k}(\tilde{y})$ is continuously differentiable on each $\gamma_{j}$, and satisfies

$$
H_{k}\left(z_{2 \jmath}\right)=0, \quad\left|H_{k}\left(z_{2 j-1}\right)\right| \leqq C h^{2} \quad \text { for } 1 \leqq j, k \leqq N,
$$

where $C$ is a positive constant which is independent of $h, j$ and $k$.

Hence we have

$$
\begin{aligned}
& \left|\int_{r_{j}} \vec{G}\left(z_{2 k}, \tilde{y}\right) \cdot\left(\vec{n}_{\tilde{y}}-\vec{m}_{\tilde{y}}^{\prime}\right) v(\tilde{y}) d \sigma_{\tilde{y}}\right| \\
& \leqq\|v\|_{\infty} \cdot \int_{r_{j}}\left|H_{k}(\tilde{y})\right| d \sigma_{\tilde{y}} \\
& \leqq C h^{3} .
\end{aligned}
$$

Considering that $\vec{n}_{\tilde{z}}^{\prime}$ (resp. $\vec{n}_{z}$ ) is a continuous (vector valued) function with respect to $\tilde{z}$ (resp. $z$ ), and that $\Omega$ is a convex domain, we have, by the intermediate value theorem, the following proposition.

Proposition 6.2. For every $\tilde{z} \in \gamma_{j}$, there exists a unique point $z \in \Gamma$, such that

$$
\vec{m}_{\tilde{z}}^{\prime}=\vec{n}_{z}
$$

Corollary 6.1. There exists a unique point $\bar{z}_{2,} \in \Gamma_{j}$ such that

$$
\vec{m}_{z_{2 j}}^{\prime}=\vec{n}_{\bar{z}_{2 j}} \text {. }
$$

Remark 6.1. Considering sinoothness of the boundary, we have by the Taylor expansion,

$$
m\left(\bar{z}_{2 \jmath} \bar{z}_{2 \jmath}\right) \leqq C h^{2},
$$

where $C$ is a positive constant which is independent of $h$.

Proposition 6.1 enables us to define a mapping from $\tilde{y} \in \tilde{\Gamma}$ to $y \in \Gamma$. Let us denote this mapping by $\tau ; \tilde{\Gamma} \rightarrow \Gamma$. Obviously this mapping is a bijection. And it is clear that $\tau$ is continuous on $\tilde{\Gamma}$ and twice continuously differentiable on each segment $\bar{z}_{k} z_{k+1}$ for $1 \leqq k \leqq 2 N-1$. Furthermore let $J(\tau)$ be its Jacobian ;

then we have

$$
\text { i.e. } J(\tau):=\frac{\partial \tau}{\partial \tilde{y}},
$$

$$
\|J(\tau)\|_{\infty} \leqq 1+C h^{2},
$$

where $C$ is a positive constant which is independent of $h$. Thus we have as follows.

Proposition 6.3. Let $v(\tilde{y})$ be a continuous function on $\tilde{\Gamma}$, then we have

$$
\left|\int_{\gamma_{j}} v(\tilde{y}) d \sigma_{\tilde{y}}-\int_{\Gamma_{j}} v \circ \tau^{-1}(y) d \sigma_{y}\right| \leqq C h^{3},
$$


where $C$ is a positive constant which is independent of $h$ and $j$.

Proof. Setting $y=\tau(\tilde{y})$, we have

$$
\begin{aligned}
I_{j} & =\int_{r_{j}} v(\tilde{y}) d \sigma_{\tilde{y}}-\int_{\Gamma_{j}} v \circ \tau^{-1}(y) d \sigma_{y} \\
& =\int_{\gamma_{j}} v(\tilde{y}) d \sigma_{\tilde{y}}-\int_{r_{j}} v \circ \tau^{-1} \circ \tau(\tilde{y}) J(\tau) d \sigma_{\tilde{y}} .
\end{aligned}
$$

By (6.4), we have

$$
\left|I_{j}\right| \leqq C h^{3} \text { 。 }
$$

Corollary 6.1 enables us to define a set of nodal points $\left\{\bar{z}_{2 k}\right\}_{k=1}^{N}$ on $\Gamma$. Here we set

$$
\phi_{k}:=\tilde{\psi}_{k} \circ \tau^{-1} \quad \text { for } \quad 1 \leqq k \leqq 2 N .
$$

These functions possess the following properties.

Proposition 6.4. Let $\left\{\psi_{k}\right\}$ be defined by (6.6), then we have

(i) $\phi_{k} \in C^{0}(\Gamma)$ for $1 \leqq k \leqq 2 N$,

(ii) $\phi_{k}\left(\bar{z}_{j}\right)=\delta_{k, j}$ for $1 \leqq k, j \leqq 2 N$, where $\bar{z}_{2 j-1}$ coincides with $z_{2 j-1}$ for $1 \leqq j \leqq N$,

(iii) $\operatorname{supp} \phi_{k}=\overbrace{\bar{z}_{k-2}} \bar{z}_{k+2}$ for $1 \leqq k \leqq 2 N$,

(iv) $\left.\phi_{k}\right|_{\widehat{\bar{z}}_{\bar{z}} \bar{z}_{j+1}} \in C^{2}\left(\widetilde{\bar{z}}_{j} \bar{z}_{j+1}\right)$ for $1 \leqq k, j \leqq 2 N$.

Let $V_{h}^{1}$ and $V_{h}^{2}$ be finite dimensional function spaces on $\Gamma$ defined by

$$
\begin{aligned}
& V_{h}^{1}:=\text { linear span of }\left\{\phi_{1}, \psi_{3}, \cdots \cdots, \phi_{2 N-1}\right\}, \\
& V_{h}^{2}:=\text { linear span of }\left\{\phi_{2}, \phi_{4}, \cdots \cdots, \phi_{2 N}\right\} .
\end{aligned}
$$

And let us define the following four collocation operators $P_{h}^{1}, P_{h}^{2}, \widetilde{P}_{h}^{2}$ and $P_{h}^{\prime}$ as follows :

$$
\begin{aligned}
& P_{h}^{1} ; C^{0}(\Gamma) \longrightarrow V_{h}^{1} \\
& U \quad U \\
& u(z) \longrightarrow \sum_{k=1}^{N} u\left(z_{2 k-1}\right) \psi_{2 k-1}, \\
& P_{h}^{2}\left(\operatorname{resp} . \tilde{P}_{h}^{2}\right) ; C^{0}(\Gamma) \longrightarrow V_{h}^{2}\left(\operatorname{resp} . \tilde{V}_{h}^{2}\right) \\
& u(z) \longrightarrow \sum_{k=1}^{N} u\left(\bar{z}_{2 k}\right) \phi_{2 k}\left(\operatorname{resp} \cdot \sum_{k=1}^{N} u\left(\bar{z}_{2 k}\right) \tilde{\psi}_{2 k}\right), \\
& \tilde{P}_{h}^{\prime} ; C^{0}(\tilde{\Gamma}) \longrightarrow \tilde{V}_{h}^{2} \\
& \Psi \quad U \\
& \tilde{u}(\tilde{z}) \longrightarrow \sum_{k=1}^{N} \tilde{u}\left(z_{2 k}\right) \tilde{\psi}_{2 k} .
\end{aligned}
$$


Hence we have the next proposition, which is similar to Proposition 3.1.

Proposition 6.5. Let $u$ be a function which belongs to $C^{2}(\Gamma)$. Then we have

$$
\begin{gathered}
\left\|P_{h}^{1} u-u\right\|_{\infty} \leqq C h^{2}, \\
\left\|P_{h}^{2} u-u\right\|_{\infty} \leqq C h^{2},
\end{gathered}
$$

where $C$ is a positive constant which is independent of $h$.

Its proof is just the same with that of Proposition 3.1. We only apply the Taylor expansion to estimate them. But here we must pay attention to the fact that a function $\phi_{2 k} \mid \Gamma_{j}$ is a piecewise $C^{2}$-function on each $\Gamma_{j}$.

Next we give the proofs of Lemma 5.1 and Proposition 5.2.

Proof of Lemma 5.1. Set

$$
a_{i, j}=\delta_{i, j}+\lim _{x \rightarrow \bar{z}_{2 i}} \int_{\Gamma} \vec{G}(x, y) \cdot \vec{n}_{y} \psi_{2 j} d \sigma_{y}
$$

for $1 \leqq i, j \leqq N$. Then we have, by Lemma 3.1 and Assumption 5.1 , the following estimates:

$$
\begin{aligned}
& -C_{1} h \leqq a_{i, j} \leqq-C_{2} h \quad \text { for } 1 \leqq i, j \leqq N, \quad i \neq j, \\
& C_{3} \leqq a_{i, i} \leqq C_{4} \quad \text { for } \quad 1 \leqq i \leqq N,
\end{aligned}
$$

where $C_{1}, C_{2}, C_{3}$ and $C_{4}$ are positive constants which are independent of $h$. Hence by (5.16) and (6.9), we have

$$
\begin{aligned}
\alpha_{i, j}-a_{i, j}= & \int_{\tilde{\Gamma}} \vec{G}\left(z_{2 i}, \tilde{y}\right) \circ \vec{m}_{\tilde{y}} \tilde{\psi}_{2 j} d \sigma_{\tilde{y}}-\int_{\Gamma} \vec{G}\left(\bar{z}_{2 i}, y\right) \cdot \vec{n}_{y} \psi_{2 j} d \sigma_{y} \\
= & \int_{r_{j-1} \cup r_{j} \cup r_{j+1}} \vec{G}\left(z_{2 i}, \tilde{y}\right) \cdot\left(\vec{m}_{\tilde{y}}-\vec{m}_{\tilde{y}}^{\prime}\right) \tilde{\psi}_{2 j} d \sigma_{\tilde{y}} \\
& +\int_{r_{j-1} \cup r_{j} \cup r_{j+1}} \vec{G}\left(z_{2 \imath}, \tilde{y}\right) \cdot \vec{m}_{\tilde{y}}^{\prime} \tilde{\psi}_{2 j} d \sigma_{\tilde{y}} \\
& -\int_{\Gamma_{j-1} \cup \Gamma_{j} \cup \Gamma_{j+1}} \vec{G}\left(z_{2 i}, y\right) \cdot \vec{n}_{y} \psi_{2 j} d \sigma_{y} \\
& +\int_{\Gamma_{j-1} \cup \Gamma_{j} \cup \Gamma_{j+1}}\left(\vec{G}\left(z_{2 i}, y\right)-\vec{G}\left(\bar{z}_{2 i}, y\right)\right) \cdot \vec{n}_{y} \psi_{2 j} d \sigma_{y} .
\end{aligned}
$$

Primarily, by Proposition 6.1,

$$
\left|\int_{r_{j-1} \cup r_{j} \cup r_{j+1}} \vec{G}\left(z_{2 i}, \tilde{y}\right) \cdot\left(\vec{m}_{\tilde{y}}-\vec{m}_{\tilde{y}}^{\prime}\right) \tilde{\psi}_{2 j} d \sigma_{\tilde{y}}\right| \leqq C h^{3} .
$$

Secondly, by Proposition 6.3, 


$$
\begin{aligned}
& \mid \int_{r_{j-1} \cup \gamma_{j} \cup \gamma_{j+1}} \vec{G}\left(z_{2 i}, \tilde{y}\right) \cdot \overrightarrow{i n} \vec{y}_{\tilde{y}}^{\prime} \tilde{\psi}_{2 j} d \sigma_{\tilde{y}} \\
& -\int_{\Gamma_{j-1} \cup \Gamma_{j} \cup \Gamma_{j+1}} \vec{G}\left(z_{2 j}, y\right) \cdot \vec{n}_{y} \psi_{2,} d \sigma_{y} \mid \leqq C h^{3} .
\end{aligned}
$$

For, by the definition of $\tau$, we have

$$
\vec{G}\left(z_{2 i}, y\right) \cdot \vec{n}_{y} \psi_{2 \jmath}(y)=\vec{G}\left(z_{2 i}, y\right) \cdot \vec{m}_{\tau^{-1}(y)}^{\prime} \tilde{\psi}_{2 j^{\circ}} \tau^{-1}(y) .
$$

Finally, let $y$ be a fixed point on $\Gamma$, then a function $\vec{G}(x, y) \cdot \vec{n}_{y}$ is a Lipschitz continuous function with respect to $x \in \boldsymbol{R}^{2}$ (see Kellogg [5; ch. 6]). Recalling Remark 6.1, we have

$$
\left|\int_{\Gamma_{j-1} \cup \Gamma_{j} \cup \Gamma_{j+1}}\left(\vec{G}\left(z_{2 i}, y\right)-\vec{G}\left(\bar{z}_{2 i}, y\right)\right) \cdot \vec{n}_{y} \psi_{2 j} d \sigma_{y}\right| \leqq C h^{3} .
$$

By (6.12), (6.13), (6.14), and (6.15), we get

$$
\left|\alpha_{i, j}-a_{i, j}\right| \leqq C h^{3},
$$

where $C$ is a positive constant which is independent of $h$. And by Proposition 5.2 , we have

$$
\left|\frac{b_{i}}{N}\right| \leqq C h^{3} \text {. }
$$

Thus, by (5.18), (6.10), (6.11), (6.16) and (6.17), we get

$$
\begin{aligned}
& -C_{1}^{\prime} h \leqq c_{i, j} \leqq-C_{2}^{\prime} h \text { for } 1 \leqq i, j \leqq N \text { and } i \neq j, \\
& C_{3}^{\prime} \leqq c_{i, i} \leqq C_{4}^{\prime} \quad \text { for } 1 \leqq i \leqq N
\end{aligned}
$$

for a sufficiently large integer $N$.

Proof of Proposition 5.2. By (5.17), we have

$$
\begin{aligned}
b_{i} & =\sum_{j=1}^{N} \alpha_{i, j} \\
& =\sum_{j=1}^{N}\left(\alpha_{i, j}-a_{i, j}\right)+\sum_{j=1}^{N} a_{i, j} .
\end{aligned}
$$

On the other hand, we have

$$
\sum_{j=1}^{N} a_{i, j}=0 . \quad \text { (See Lemma 3.1.) }
$$

Thus, by (6.16), we have

$$
\begin{aligned}
\left|b_{i}\right| & \leqq \sum_{\jmath=1}^{N}\left|\alpha_{i, j}-a_{i, j}\right| \\
& \leqq N \cdot C h^{3} \\
& \leqq C^{\prime} h^{2} .
\end{aligned}
$$


Remark 6.2. Let $\tilde{K}_{h}^{\prime}$ be the minor matrix of the matrix $\tilde{K}_{h}$;

$$
\tilde{K}_{h}^{\prime}:=\left(c_{i, j}\right)_{1 \leqq i \leqq N-1} \text {. }
$$

From Lemma 5.1, we can get the same results with that of Proposition 4.2. In other words, $\tilde{K}_{h}^{\prime}$ is a regular matrix for a sufficiently large integer $N$, and we have

$$
\left\|\tilde{K}_{h}^{\prime-1}\right\|_{\infty} \leqq C \cdot \frac{1}{h},
$$

where $C$ is a positive constant which is independent of $h$.

Let us now start the error estimation for Scheme II. Let $u$ be the solution of (2.9) and (2.10), and let $\tilde{u}_{h}$ be the solution of (5.26) and (5.27). And let $\tilde{e}_{h}$ be defined by

$$
\tilde{e}_{h}:=\tilde{P}_{h}^{2} u-\tilde{u}_{h} \text {. }
$$

The following theorem holds.

Theorem 6.1. Let $\tilde{e}_{h}$ be defined by (6.19), and let $N$ be a sufficiently large integer. Then, we have

$$
\left\|\tilde{e}_{h}\right\|_{\infty} \leqq C h,
$$

where $C$ is a positive constant which is independent of $h$.

Proof. We apply the same technique as is used in the proof of Theorem 4.1. We decompose $\tilde{K}_{h} \tilde{e}_{h}$ in the following form:

$$
\tilde{K}_{h} \tilde{e}_{h}=\left(\tilde{K}_{h} \widetilde{P}_{h}^{2} u-\tilde{P}_{h}^{2} K P_{h}^{2} u\right)+\tilde{P}_{h}^{2} K\left(P_{h}^{2} u-u\right)+\left(\tilde{P}_{h}^{2} r-\tilde{P}_{h}^{\prime} \tilde{r}\right)+\left(\tilde{P}_{h}^{\prime} \tilde{r}-\tilde{r}_{h}\right) .
$$

Set (the right hand side of $(6.21)):=\left(t_{h}^{1}, t_{h}^{2}, \cdots \cdots, t_{h}^{N-1}, *\right)^{T}$. If we prove $\left|t_{h}^{k}\right|=O\left(h^{2}\right)$ for $1 \leqq k \leqq N-1$, and if we prove

$$
\left|\int_{\tilde{\Gamma}} \tilde{e}_{h} d \sigma_{\tilde{y}}\right|=O\left(h^{2}\right),
$$

then we can immediately get the conclusion. (See the proof of Theorem 4.1.) Hence we are sufficient to estimate $\left.\tilde{K}_{h} \tilde{e}_{h}\right|_{\tilde{z}=z_{2}}$. By (5.18), (5.26) and (6.17), we have

$$
\begin{aligned}
\left|\left(\tilde{K}_{h} \tilde{P}_{h}^{2} u-\tilde{P}_{h}^{2} K P_{h}^{2} u\right)\left(z_{2 k}\right)\right| & =\left|\sum_{k=1}^{N} c_{k, j} u\left(\bar{z}_{2 k}\right)-\sum_{k=1}^{N} a_{k, j} u\left(\bar{z}_{2 k}\right)\right| \\
& \leqq\|u\| \|_{\infty} \sum_{k=1}^{N}\left|c_{k, j}-a_{k, \jmath}\right| \\
& \leqq C h^{2} .
\end{aligned}
$$

By Proposition 6.5, we have

$$
\left|\widetilde{P}_{h}^{2} K\left(P_{h}^{2} u-u\right)\left(z_{2 k}\right)\right| \leqq C h^{2} .
$$

Furthermore we have 


$$
\begin{aligned}
\left(\tilde{P}_{h}^{2} r-\tilde{P}_{h}^{\prime} \tilde{r}\right)\left(z_{2 k}\right)= & \left(r\left(\bar{z}_{2 k}\right)-r\left(z_{2 k}\right)\right)+\int_{\tilde{T}^{\prime}} g\left(z_{2 k}, y\right)\left(q-P_{h}^{1} q\right) d \sigma_{y} \\
& +\int_{\Gamma} g\left(z_{2 k}, y\right) P_{h}^{1} q d \sigma_{y}-\int_{\tilde{\Gamma}} g\left(z_{2 k}, \tilde{y}\right) \tilde{P}_{h}^{1} q d \sigma_{\tilde{y}}
\end{aligned}
$$

Considering that $r(z)$ is a Lipschitz continuous function with respect to a variable $z \in \boldsymbol{R}^{2}$ (see Kellogg [5; ch.6]), and recalling Remark 6.1, then we have

$$
\left|z\left(\bar{z}_{2 k}\right)-r\left(z_{2 k}\right)\right| \leqq C h^{2} \text {. }
$$

By Proposition 6.3 and Proposition 6.5, we get

$$
\left|\left(\tilde{P}_{h}^{2} r-\tilde{P}_{h}^{\prime} \tilde{r}\right)\left(z_{2 k}\right)\right| \leqq C h^{2} 。
$$

Therefore by (6.22), (6.23), (6.24) and (6.25), we get

$$
\left|t_{h}^{k}\right| \leqq C h^{2} \quad \text { for } \quad 1 \leqq k \leqq N-1 \text {. }
$$

On the other hand, by (2.10) and (5.27), we have

$$
\begin{aligned}
\left|\int_{\tilde{\Gamma}} \tilde{e}_{h} d \sigma_{\tilde{y}}\right| & \leqq\left|\int_{\Gamma}\left(u-P_{h}^{2} u\right) d \sigma_{y}\right|+\left|\int_{\Gamma} P_{h}^{2} u d \sigma_{y}-\int_{\tilde{\Gamma}} \tilde{P}_{h}^{2} u d \sigma_{\tilde{y}}\right| \\
& \leqq C h^{2} .
\end{aligned}
$$

Hence we can get the conclusion.

Let us define a interpolation operator $I_{h}$ from $\tilde{V}_{\bar{h}}^{2}$ into $C^{0}(\Gamma)$ as follow:

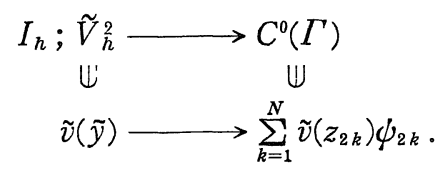

Theorem 6.1 is equivalent to the next theorem.

Theorem 6.2. Let $u$ be the solution of (2.9) and (2.10), and let $\tilde{u}_{h}$ be the solution of (5.26) and (5.27). Then we have, for a sufficiently large integer $N$,

$$
\left\|u-I_{h} u_{h}\right\|_{\infty} \leqq C h,
$$

where $C$ is a positive constant which is independent of $h$.

Proof. Obviously we have

$$
u-I_{h} \tilde{u}_{h}=\left(u-P_{h}^{2} u\right)+I_{h} \tilde{e}_{h} 。
$$

From Proposition 6.5 and Theorem 6.1, we can get the conclusion. (q.e.d.)

\section{§ 7. Conclusion}

In this paper, we give two numerical schemes and give the convergence 
theorems for them. (See Theorem 4.1 and Theorem 6.2.) Since the coefficient matrices are ill-conditioned for both schemes, we only get the low-order error estimates. In other words, the results of this paper are the convergence theorems of $O(h)$, though the truncation errors are of $O\left(h^{2}\right)$. But many numerical datas, which are done in engineerings, suggest that we may prove the convergence theorems of $O\left(h^{2}\right)$ for our schemes. To this point, details are shown in Hayakawa-Iso [4].

\section{Acknowledgement}

The author wishes to express his sincere acknowledgement to Prof. S. Hitotumatu and Prof. M. Yamaguti for their constant encouragements for the author. And the author also wishes to express his gratitude to Prof. K. Hayakawa for his constant encouragements and his fruitful suggestions.

\section{References}

[1] Arnold, D. N., Wendland, W.L., On the Asymptotic Convergence of Collocation Methods, Math. Comp., 41 (1983), 349-381.

[2] Arnold, D. N., A Spline-Trigonometric Method and an Exponentially Convergent Boundary Integral Method, Math. Comp., 41 (1983), 383-397.

[3] Brebbia, C.A., The Boundary Element Method for Engineers, Pentech Press, 1978.

[4] Hayakawa, K., Iso, Y., High-order Uniform Convergence Estimates for Boundary Solutions for Laplace's Equation, (in preparation).

[5] Kellogg, O.D., Foundations of Potential Theory, Dover, 1953. 
